\title{
COMPUTATIONAL MODELING AND EXPERIMENTAL CHARACTERIZATION OF MARTENSITIC TRANSFORMATIONS IN NICOAL FOR SELF-SENSING MATERIALS
}

\author{
T. A. Wallace ${ }^{1}$, V. I. Yamakov ${ }^{1,2}$, J. D. Hochhalter ${ }^{1}$, W. P. Leser ${ }^{1}$, J. E. Warner ${ }^{1}$, J. A. Newman ${ }^{1}$, \\ G. P. Purja Pun ${ }^{3}$, Y. Mishin ${ }^{3}$ \\ ${ }^{1}$ NASA Langley Research Center, Hampton, VA 23681, USA \\ ${ }^{2}$ National Institute of Aerospace, Hampton, VA 23666, USA \\ ${ }^{3}$ George Mason University, Fairfax, VA 22030, USA \\ Keywords: Keyword1, Keyword2, Keyword3, etc.
}

\begin{abstract}
Fundamental changes to aero-vehicle management require the utilization of automated health monitoring of vehicle structural components. A novel method is the use of self-sensing materials, which contain embedded sensory particles (SP). SPs are micron-sized pieces of shape-memory alloy that undergo transformation when the local strain reaches a prescribed threshold. The transformation is a result of a spontaneous rearrangement of the atoms in the crystal lattice under intensified stress near damaged locations, generating acoustic waves of a specific spectrum that can be detected by a suitably placed sensor. The sensitivity of the method depends on the strength of the emitted signal and its propagation through the material. To study the transition behavior of the sensory particle inside a metal matrix under load, a simulation approach based on a coupled atomistic-continuum model is used. The simulation results indicate a strong dependence of the particle's pseudoelastic response on its crystallographic orientation with respect to the loading direction and suggest possible ways of optimizing particle sensitivity. The technology of embedded sensory particles will serve as the key element in an autonomous structural health monitoring system that will constantly monitor for damage initiation in service, which will enable quick detection of unforeseen damage initiation in real-time and during onground inspections.
\end{abstract}

\section{Introduction}

The concept aerospace vehicles that will enable future space missions must be designed for service conditions that may not be repeatable in laboratory environments and may experience loads that are not foreseen during the design phase. Also, current design approaches rely heavily on empirical data, which do not exist for the multifunctional next-generation materials that will be required to enable future vehicles, again contributing to increased uncertainty. Currently, approaches ensuring safe operation in highly uncertain conditions come at the cost of over design, over conservative inspection, and unnecessarily frequent part replacement. Further, certification of such parts often requires costly test programs.

The technology discussed is the utilization of sensory particles made of shape memory alloy (SMA) and embedded within an aerospace structural component to automatically monitor specific structural components while in service [1]. The embedded sensory particles will serve as the key element in an autonomous structural health monitoring system that will constantly and 
automatically monitor for damage initiation. Automated monitoring will enable quick detection of unforeseen structural failure in real-time and during on-ground inspections.

This study demonstrates the suitability of SMA particles as fatigue crack sensors in aluminum alloys. A strain-induced phase transformation will be triggered in the SMA material by the high strains that occur near a fatigue crack tip, and the resulting AE signal can be detected using commercially-available AE monitoring technology. The relationship between the strain-induced martensitic transformation and the generation of acoustic emission (AE) was investigated experimentally, and through atomistic simulations for the Ni-Co-Al SMA system. A multiscale atomistic-continuum model was developed to evaluate the acoustic response of an embedded SMA particle in aluminum metal matrix under different loading conditions.

\section{Experimental Procedure}

Ni-Co-Al was produced by melting the constitutive elements in a vacuum induction furnace. The atomic percent ratio of the constituents was: Ni:35, Al:29, and Co:36. The casting was homogenized at $1350^{\circ} \mathrm{C}$ for 100 hours and furnace cooled. Samples were cut from the casting using EDM. A solution heat treatment was performed at $1350^{\circ} \mathrm{C}$ in a custom rapid quench furnace. The samples were annealed in an argon gas inert environment and quenched in liquid argon (-187C). Specimens from the alloy system underwent additional heat treatments ranging from $325 \mathrm{C}$ to $600 \mathrm{C}$ in order to simulate potential embedding processes. This was done by heating the specimens in a vacuum sealed quartz tube for 1 hour followed by a water quench.

The compression tests were carried out in displacement control at a displacement rate of $4.2 \times 10^{-4}$ $\mathrm{mm} / \mathrm{sec}$. The specimens were cylindrical approximately $1 \mathrm{~mm}$ tall with a $2.5 \mathrm{~mm}$ diameter. Due to the small size of the specimens, direct strain measurement was not practical. Therefore, strain data was estimated using machine displacement, compensating for test machine compliance.

AE events were captured using Digital Wave B-1025 piezoelectric sensors. The captured signals were amplified and recorded at a sampling rate of $10 \mathrm{MHz}$. AE measurements were aquired indirectly through a sensor attached to the lower platen in close proximity to the sample.

\section{Experimental Results}

An example of a stress-strain curve for bulk SMAs is shown in Fig. 1(a). The occurrence of AE signals are indicated as triangles along the stress-strain curve. In order to correlate the straininduced transformation with recorded AE events across multiple samples, a transformation stress ratio, $\sigma_{T}=\sigma_{A E} / \sigma_{c r}$, was defined for each captured event, where $\sigma_{\mathrm{AE}}$ is the stress level at which the AE event was captured, and $\sigma_{\mathrm{cr}}$ is the critical stress for transformation of the SMA as determined from the stress-strain response of each specimen. The probability densities of $\sigma_{\mathrm{T}}$ across all samples ( 2 samples, cycled 3 times each, resulting in approximately 2000 AE events) are shown in Fig. 1(b). The strain-induced phase transformation is clearly indicated by a sharp increase in the AE activity near $\sigma_{\mathrm{T}}=1$. The reverse transformation that occurs upon unloading is also seen at $\sigma_{\mathrm{T}}=0.4$. These results clearly demonstrate a correlation between the strain-induced phase transformation and AE activity. 

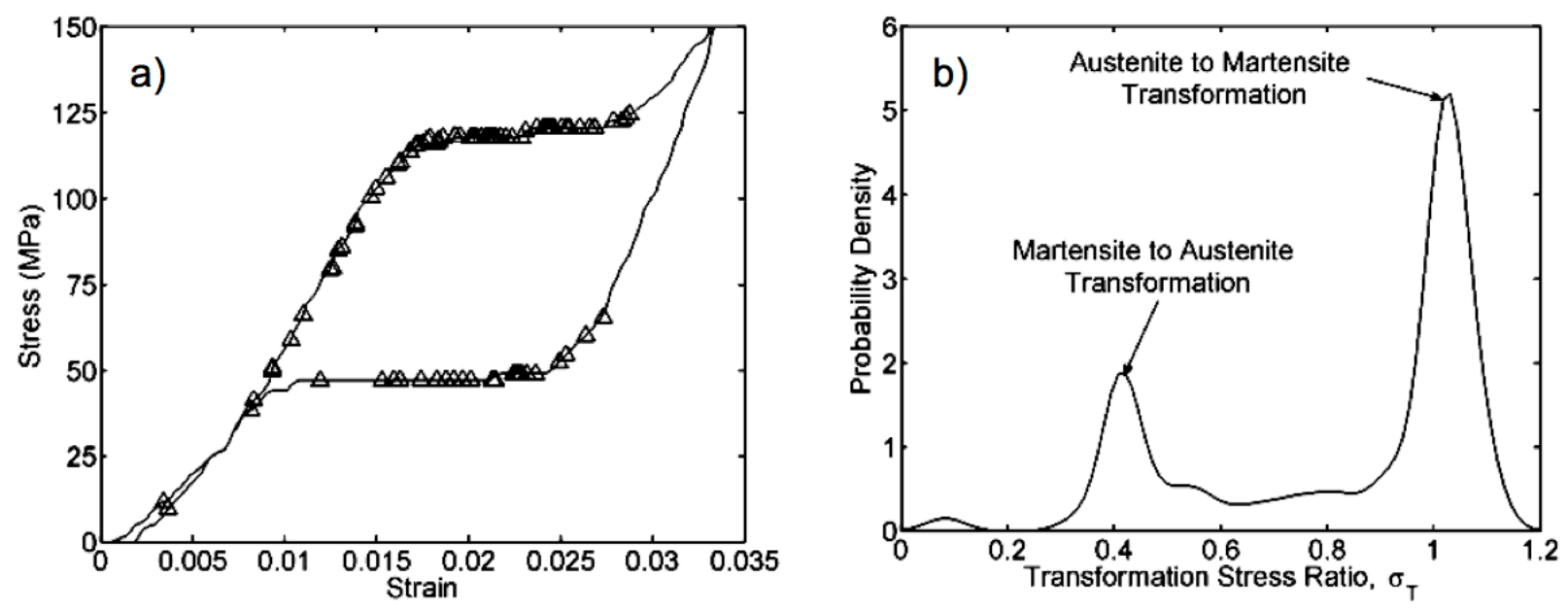

Figure 1. a) Stress vs strain curve, and b) Transformation stress ratio of AE events during compression tests on Ni-Co-Al specimens.

\section{Multiscale Simulation Model}

The multiscale model employs the embedded-statistical-coupling method (ESCM), described in detail in ref. [2]. The method consists of an atomistic domain simulated by molecular dynamics (MD), embedded in a larger continuum domain simulated by an explicit dynamic finite element (FE) integration method. The geometry and the dimensions of the overall system model are given in Fig. 2. For this study, the atomistic domain represented the SMA particle of composition given in atomic percent: Ni:34.8, Al:28.8, and Co:36.4, which closely matched the experimental alloy system. This composition was prepared separately using semi-grand canonical Monte Carlo (SGMC) method [3] utilizing the interatomic potential for Ni-Co-Al ternary system by Ganga et al. [4]. The FE domain represented the surrounding Al matrix as an elastic membrane.

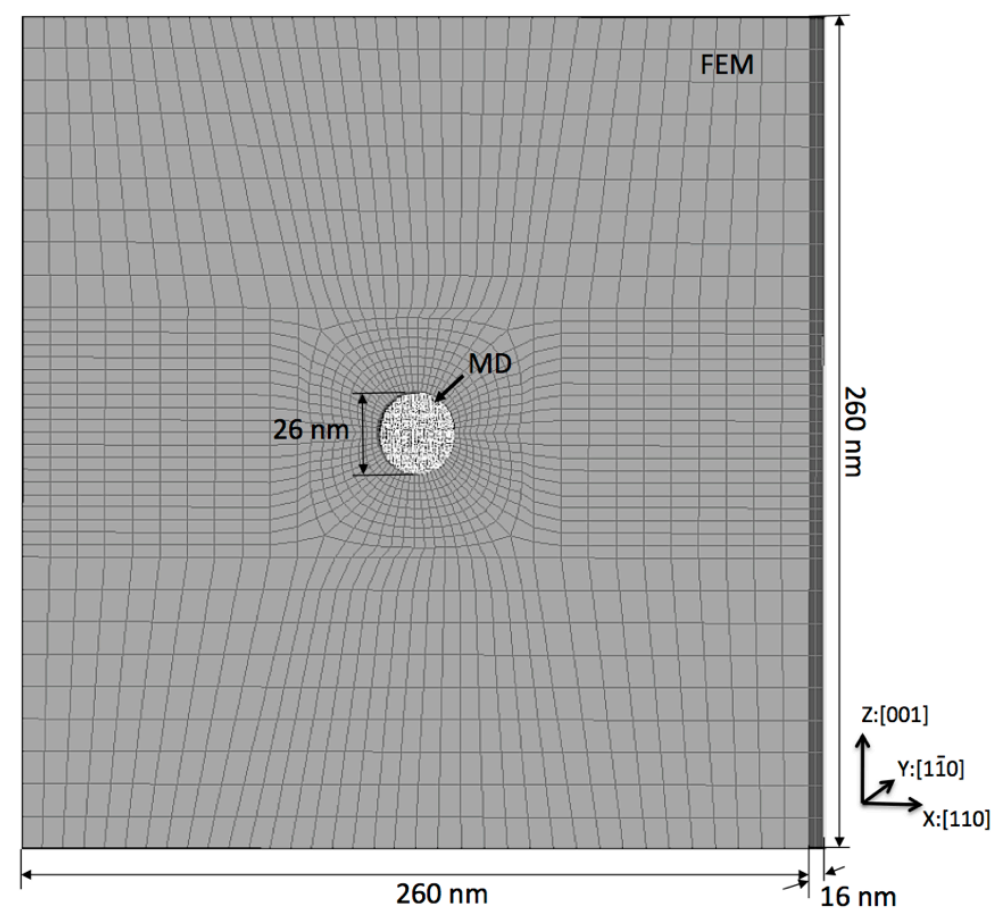

Figure 2. Multiscale model of an embedded SMA particle, represented by $\mathrm{MD}$, into an Al metal matrix, represented by FEM. 
The MD method evolves the system by solving the Newtonian equation of motion defined for each individual atom of the SMA material as

$$
\mathbf{m} \ddot{\mathbf{r}}=\mathbf{f}+\mathbf{p}
$$

Here $m$ is the mass of the atom at a position vector $\mathbf{r}, \mathbf{f}$ is the interatomic force exerted on the atom by its neighbors, and $\mathbf{p}$ is a time-dependent external to the MD domain force per atom.

Similarly, the explicit FE method solves the equation defined for each FE node as

$$
\mathbf{M u ̈}=\mathbf{F}+\mathbf{P},
$$

where $\mathbf{M}$ is the mass matrix, $\mathbf{u}$ is the nodal displacement, $\mathbf{F}$ is the internal element force vector, and $\mathbf{P}$ is a time-dependent applied external force.

At the MD/FE interface the two domains are linked statistically through the system

$$
\mid \begin{aligned}
& \mathbf{u}=\langle\mathbf{r}\rangle \\
& \dot{\mathbf{u}}=\langle\dot{\mathbf{r}}\rangle
\end{aligned},
$$

and

$$
\mathbf{p}=\mathbf{F}(\mathbf{u}, \dot{\mathbf{u}}) / n
$$

Here \langle\rangle indicates averaging over time and volume of $n$ number of atoms contained in a predefined atomistic subdomain at the MD/FE interface related to a certain interface FE node. Equation (2a) defines an interface boundary value problem for Eq. (1b) in the FE domain, which solution gives the reaction force $\mathbf{F}(\mathbf{u}, \dot{\mathbf{u}})$ for the interface nodes in Eq. (2b). This force is uniformly distributed as an external force $\mathbf{p}$ over the interface atoms of the MD domain through Eq. (1a). An iterative exchange of continuously updated displacements and reaction forces between the MD and FEM simulations establishes a dynamic force/displacement balance and continuity between the FEM and the MD domains.

The advantages of using statistical coupling (Eq. 2) are two-fold: first, it allows for large atomistic domains ( $>10^{6}$ atoms) to be embedded into micron size FEM domains because of the dramatic reduction of the degrees of freedom at the $\mathrm{MD} / \mathrm{FE}$ interface; second, elevated temperatures can readily be simulated because the motion of individual atoms is not constrained to strictly follow the FEM interface displacements, but only their statistical value, which allows for the thermal vibrations to be unconstrained.

\section{Simulation Results}

$\underline{\text { Single Crystal Ni-Co-Al SMA properties }}$

The pseudoelastic behavior of the simulated single crystal Ni-Co-Al system was studied by applying cyclic tension/compression load, first in the $z$ :[001] direction, and then in the $x:[110]$ direction of the $\mathrm{B} 2$ phase while the stress in the other directions was kept at zero. To ensure that the system at zero pressure was in the stable austenite phase the temperature of the simulation was set at $\mathrm{T}=550 \mathrm{~K}$, which is $25 \mathrm{~K}$ above the austenite finish temperature, $A_{\mathrm{f}}=525 \mathrm{~K}$, estimated in a separate simulation. Figure 3 shows that the strain increase of up to $10 \%$ in the [001] load, and up to $4 \%$ in the [110] load, was fully recoverable upon unloading, which demonstrated the pseudoelastic effect. The strain-stress hysteresis loops were smaller when a 
twinned martensite was formed, namely, in compression in the [001] direction, and in tension in the [110] direction. Tension in the [001] direction or compression in the [110] direction produced detwinned martensite, resulting in a higher pseudoelastic strain. In addition, the transformation required higher tensile stress in the [110] direction than in the [001] direction. This is consistent with the Bain mechanism of lattice transformation from $\mathrm{B} 2$ to $\mathrm{L} 1_{0}$ phase, which takes place through simultaneous elongation along the [001] direction and contraction in the two perpendicular $<110>$ directions. Thus, the transformation depends on the principle stress along the [001] direction, which is maximal when this is the direction of the applied load.

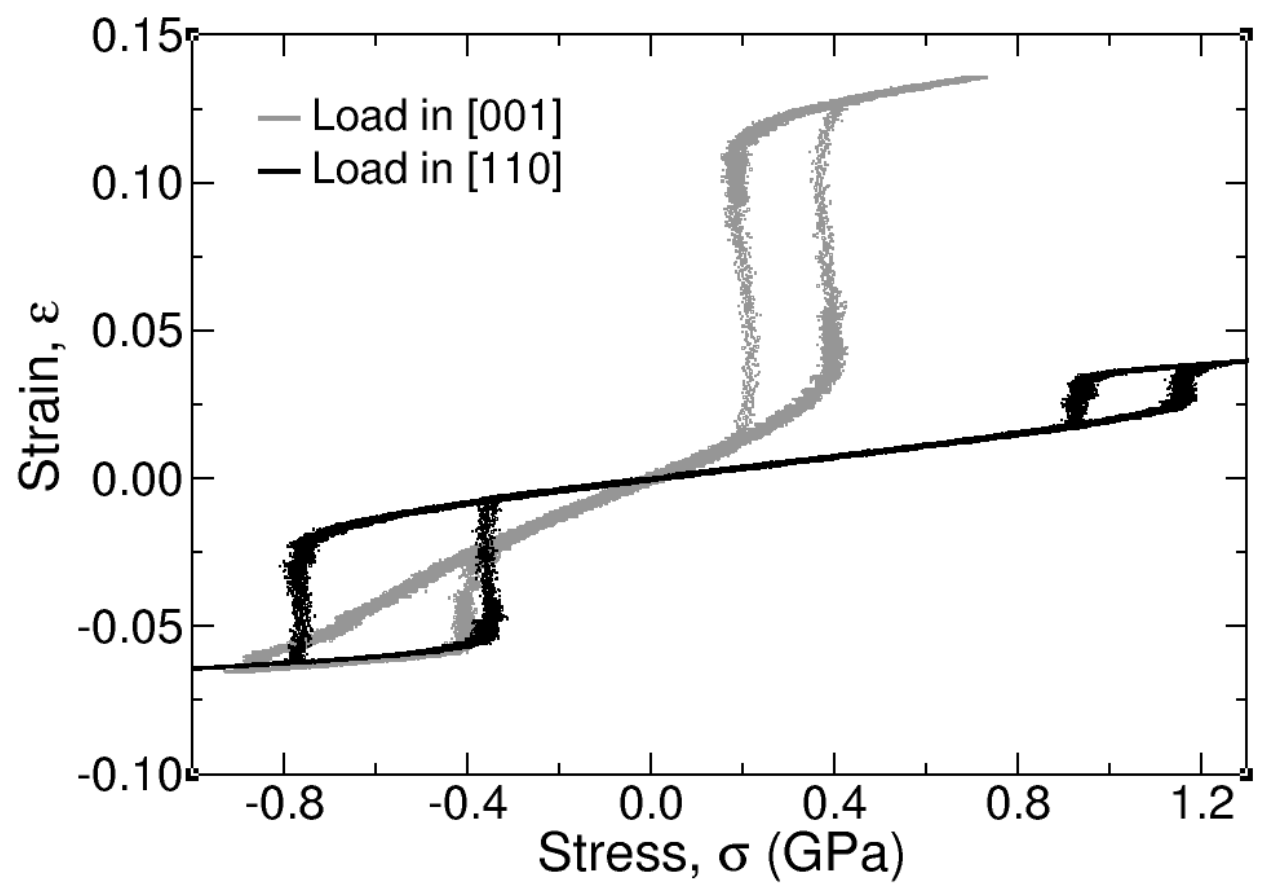

Figure 3. Stress vs strain curve during tension-compression cyclic loading of a simulated Ni-Co-Al system at $\mathrm{T}=550 \mathrm{~K}$.

\section{SMA Particle Embedded in Al Matrix Under Shockwave Loading}

To study the behavior of the SMA particle embedded in Al matrix under shock loading, the multiscale model in Fig. 2 was loaded by applying a constant speed of elongation, equal to 20.8 $\mathrm{m} / \mathrm{s}$ on one of the sides of the system for $100 \mathrm{ps}$, which resulted in $8 \%$ overall elongation. This created a shock wave starting from one side of the system and traveling with the speed of sound $(5500 \mathrm{~m} / \mathrm{s}$ for Al) towards the opposite side. As shown in Fig. 4, when the upper side is loaded, the shock wave propagates towards from top to bottom (Figs. 4a,b) and intercepts with the particle from the [001] direction. Alternatively, when the right side is loaded (Figs. 4c,d), the shock wave propagates from right to left and intercepts the particle from the [110] direction. The state of the SMA particle during the simulation was monitored through the overall virial atomic stress [5], in the particle volume, and by following the crystallographic state of the atoms determined through common neighbor analysis (CNA) [6]. CNA identifies the austenite B2 phase as atoms in the bcc crystallographic state, while the martensite $\mathrm{L} 1_{0}$ phase is identified as atoms in a distorted fcc state which may contain twins appearing as lines of hcp atoms.

In both cases of loading directions, the particle did not transform immediately when reached by the shock wave (Figs. 4a,c), but only after the load reached a certain threshold level (Figs. 4b,d). 
At that instant, the front of the particle, facing the shock wave, transformed first, with the transformation propagating further into the particle volume. Note that in the case of the [001] wave, the strain profile at the front of the particle formed a double-leaf shape (Fig. 4b), indicating a relieved strain directly in front of the particle, which was not found in Fig. 4d, for the [110] wave front. This stress relief is a result of the non-linear pseudoelastic response of the particle, which is strongest in the [001] direction. In this case of the [110] direction, the particle underwent only partial transformation, which could be explained with the higher transformation stress needed in this direction as compared with the [001] direction.

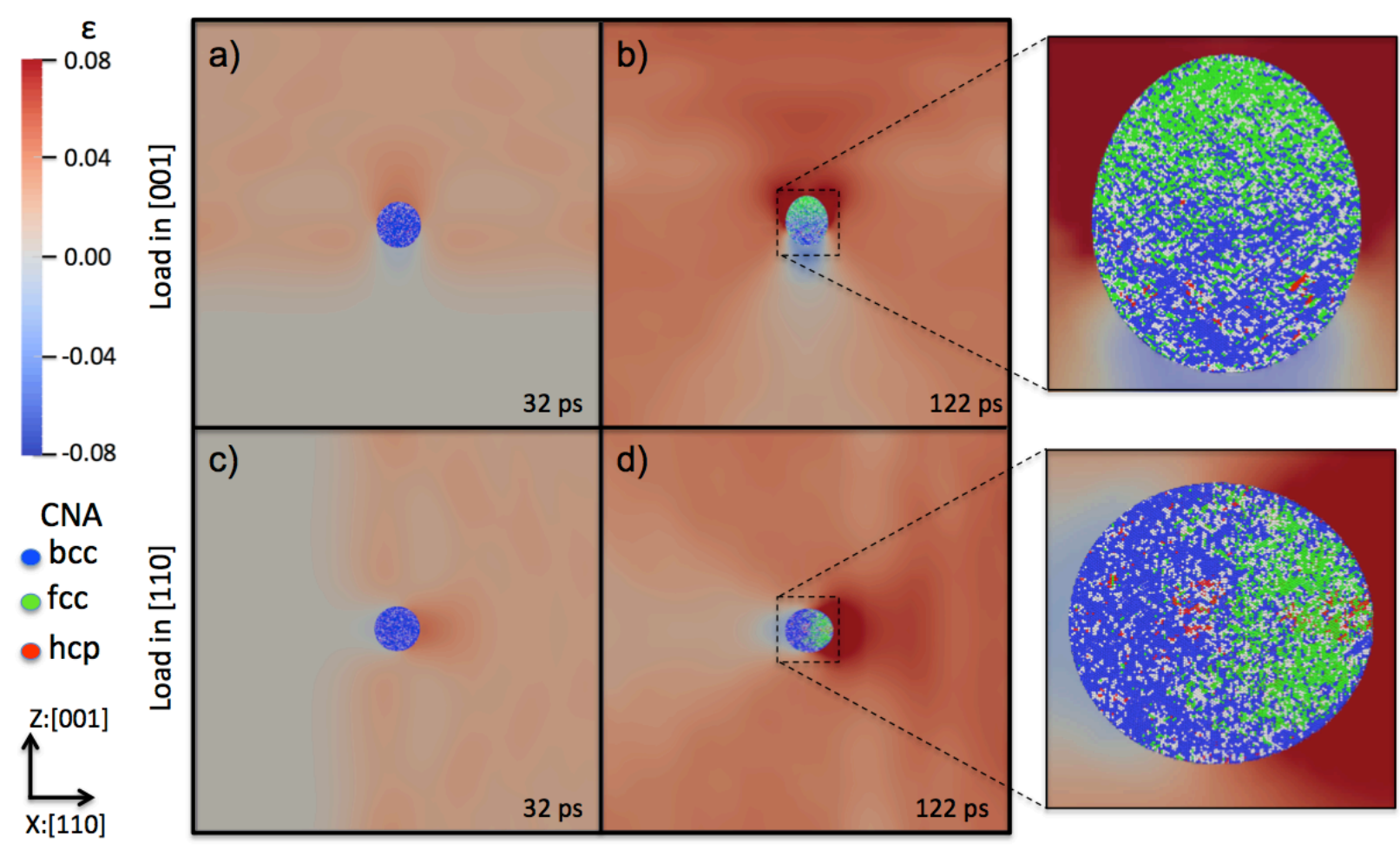

Figure 4. Series of snapshots taken at the indicated times of the multiscale model during shock wave propagation generated in the $(\mathrm{a}, \mathrm{b}) z:[001]$ direction (vertical), and (c,d) $x:[110]$ direction (horizontal). The color of the centered circular particle, also zoomed in the insets, represents the crystallographic state as defined by the CNA as indicated in the figure, while the color of the surrounding continuum represents the level of strain.

To isolate the effect of the martensitic transformation on the stress inside the SMA particle, a comparison is used with a reference inert particle that does not experience transformation, but has the same elastic properties as the SMA in its austenite, pre-transformed, phase. Figure 5(a) shows the difference in the virial stress, $\Delta \sigma$, between the transformed SMA particle and the reference inert particle subject to the same loading regime, for the two loading directions, [001] and [110]. The plotted curves indicate that when the shock wave approaches the SMA particle from the [001] direction, the stress response inside the particle is much stronger, compared to the [110] incident direction.

Figure 5(b) shows the content of the B2 phase (number of bcc atoms) compared to the $\mathrm{L} 1_{0}$ phase (fcc + hcp atoms) during wave propagation. The peak of the transformation occurs at around 
$t=120 \mathrm{ps}$. The amount of the martensite phase, formed during the [001] loading is approximately $60 \%$ larger compared to the [110] loading ( $c=0.5$ vs. $c=0.3$, respectively). In addition, there is a noticeable increase of the hcp atoms, indicating the formation of twinned martensite during the [110] loading. By contrast, the [001] loading forms detwinned martensite. Twinning accommodates the transformation strain and effectively softens the particle, relieving the internal stress.
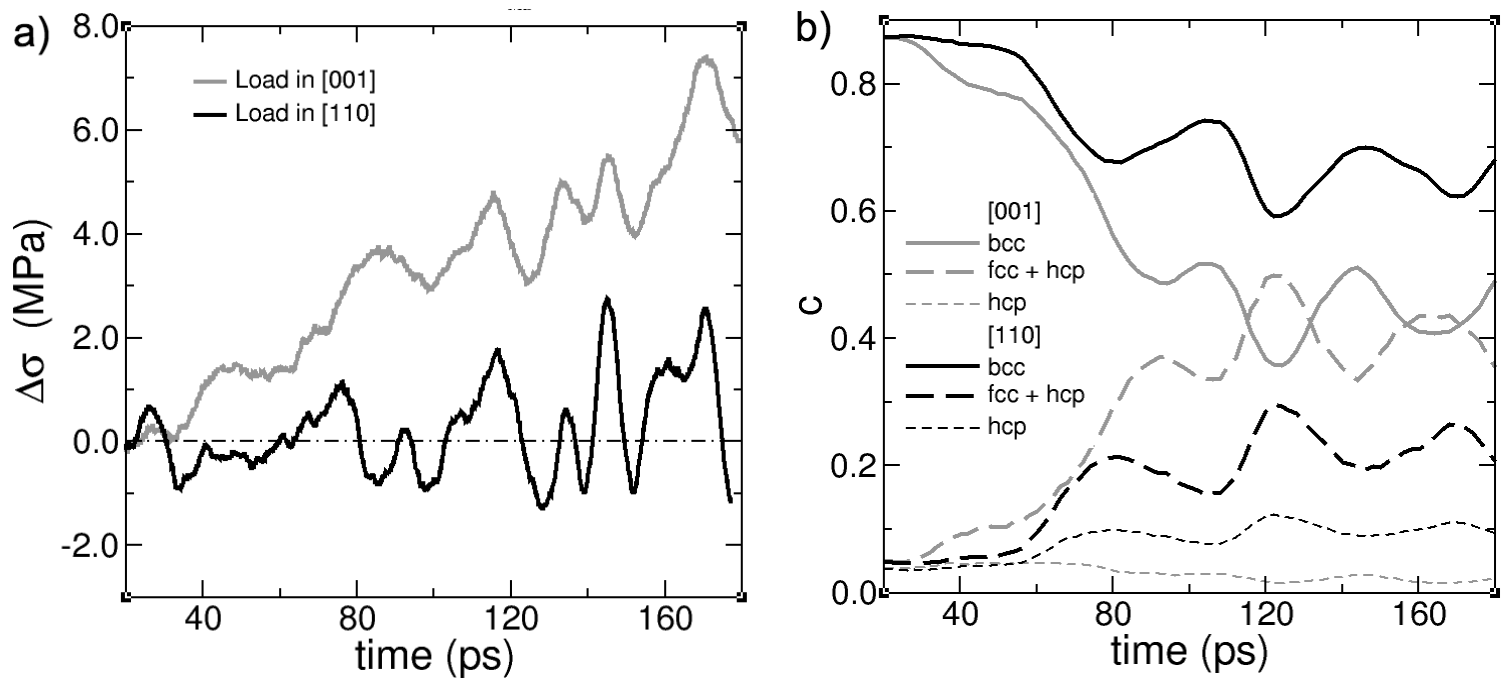

Figure 5. (a) Differential virial stress, $\Delta \sigma$, between the transformed SMA particle and the reference inert particle for the two loading directions, [001] and [110]; (b) Content of the B2 (bcc), L1 $1_{0}$ (fcc+hcp), and $\mathrm{L} 1_{0}$ twins (hcp) atoms in the SMA particle with time.

\section{Conclusion}

In summary, an experimental and a multiscale atomistic-continuum simulation studies were performed to examine the response of a small SMA particle of Ni-Co-Al alloy embedded in Al matrix to a propagating mechanical shock wave. The study was motivated by a possible implementation of such structures for early damage detection in materials for aerospace applications.

The experimental results confirmed the occurrence of martensitic transformation inside the NiCo-Al SMA during fatigue tests accompanied by detectable acoustic emission. The results of the multiscale simulation showed that the response of an SMA particle, when embedded in Al metal matrix, strongly depends on its crystallographic orientation with respect to the shock wave front. The particle is more sensitive when the wave front propagation direction coincides with the [001] crystallographic axis as compared to the [110] axis. The decreased sensitivity in the [110] direction is due to two factors. First, the Bain mechanism of transformation favors loads along the [001] direction showing a stress - strain pseudoelastic hysteresis at much lower tensile stresses (between 0.2 and $0.4 \mathrm{GPa}$ in tension), compared to the loads in the [110] direction (between 0.9 and $1.2 \mathrm{GPa}$ ). As a result, the transformed volume under shock waves of the same amplitude was found to be about $60 \%$ larger in the case of [001] wave propagation than for the [110] wave propagation. Second, in the case of the [110] direction, the transformation produced 
strongly twinned martensite, whereas the [001] wave produced only detwinned martensite. As a result, twinning softened the particle and weakened its acoustic emission in the matrix.

\section{Acknowledgements}

V. Yamakov is sponsored through cooperative agreement NCC-1-02043 with the National Institute of Aerospace. G. P. Purja Pun and Y. Mishin were supported by the National Aeronautics and Space Administration through the NASA Langley Research Center (cooperative agreement NRA \# NNX08AC07A).

\section{References:}

1. T.A. Wallace, S.W. Smith SW, R.S. Piascik, M.R. Horne, P.L. Messick, J.A. Alexa, E.H. Glaessgen, B.T. Hailer "Strain-Detecting Composite Materials" (Patent Application Publication, Pub. No. US2010/0190026 A1, July 29, 2010).

2. E. Saether, V.I. Yamakov, E.H. Glaessgen, "An Embedded Statistical Coupling Method for Coupling Molecular Dynamics with Finite Element Analyses," Int. J. Numer. Meth. Engng. 78 (2009), 1292-1319.

3. Daan Frenkel, and Berend Smit, Understanding Molecular Simulation: From Algorithms to Applications (Academic Press, San Diego, second edition, 2002), 662.

4. G.P. Purja Pun, V.I. Yamakov, Y. Mishin, "Interatomic Potential for the Ternary Ni-AlCo System and Application to Atomistic Modeling of the B2-L1 ${ }_{0}$ Martensitic Transformation," Modeling Simul. Mater. Sci. Eng. (submitted); NIST Interatomic Potentials Repository: http://www.ctcms.nist.gov/potentials/

5. J. Cormier, J.M. Rickman, and T.J. Delph, "Stress Calculation in Atomistic Simulations of Perfect and Imperfect Solids," J. Appl. Phys. 89 (2001), 99-104.

6. A.S. Clarke, H. Jonsson, "Structural Changes Accompanying Densification of Random Hard-Sphere Packings," Phys. Rev. E, 47 (1993), 3975. 